\title{
Design of Panoramic Ionospheric Recorders
}

\author{
L. H. Heisler and L. D. Wilson \\ Contribution from Radio Research Board Laboratory, Electrical Engineering School, University of Sydney, Australia
}

(Received January 3, 1961; revised May 26, 1961)

\begin{abstract}
The design of ionosondes and the many electronic techniques used in the panoramic type are considered. A complete ionosonde which is readily transportable, economic to construct, and has the additional facility of electronic scan over any portion of the frequency range 1.0 to 20 megacycles per second is described briefly.
\end{abstract}

\section{Introduction}

It is becoming increasingly obvious that one of the most valuable ground based instruments for upper atmosphere research is the ionosonde recorder. Electronic computers and simplified reduction techniques such as those of Kelso [1954], or more recently Schmerling and Ventrice [1959], enable electron density distributions to be readily obtained from the corresponding $h^{\prime} f$ records, and recent efforts to tabulate these results on a worldwide basis are providing invaluable information on global ionospheric morphology. The need for more frequent ionospheric samplings is suggested by the presence of traveling ionospheric disturbances described by Munro [1950] and Heisler [1958]. This work indicates that the ionosphere is a dynamic medium, in which almost continuous changes in ion density are occurring. Furthermore, recent discoveries of a traveling disturbance - $E_{s}$ relationship by Heisler and Whitehead [1960] and the sporadic nature of $E_{s}$ itself, suggest that spaced ionosonde recorders are essential to study growth of $E_{s}$ patches and mechanics of ionospheric disturbances.

Such recorders are, of necessity, very complex, and involve many diverse electronic techniques. The panoramic type recorder as originally described by Sulzer [1949] is now invariably used in modified forms such as that due to Heisler [1955], or as in existing American type C4 recorders [Brown, 1959]. However, the equipment is usually bulky and expensive. Also there seems to be lack of detailed design information available on this type of recorder.

This paper considers ionosonde characteristics necessary to satisfy recent research requirements. Design principles of the panoramic type ionosonde are discussed in detail and finally a new recorder is described which is readily transportable and economic to construct, possessing the additional facility of electronic scan over any portion of the frequency range 1 to $20 \mathrm{Mc} / \mathrm{s}$.

\section{Basic Description}

The basic ionosonde recorder consists of a receiver tuned synchronously to a transmitter which scans over the frequency range $f_{0}$ to $f_{1} \mathrm{Mc} / \mathrm{s}$ in the scan period $T$ sec, at the scan rate of $S$ scans per hour. The transmitter is pulsed, the pulse length being $t \mu$ sec at a repetition frequency of $F \mathrm{c} / \mathrm{s}$, where $F$ usually is equal to the local a-c supply frequency. The directly received pulse and echoes are displayed on one axis of a cathode ray oscillograph display calibrated in height, and a calibrated frequency scale is provided either by movement of the height scale across the axis of movement of the recording film or by movement of the film itself.

In older types of recorders synchronization between transmitter and receiver was maintained by the use of cams and band switching. In the panoramic type recorder this is avoided by covering the frequency range in a single sweep. This is achieved by heterodyning an oscillator continuously variable from $f_{0}+f_{x} \mathrm{Mc} / \mathrm{s}$ to $f_{1}+f_{x} \mathrm{Mc} / \mathrm{s}$ with an $f_{x} \mathrm{Mc} / \mathrm{s}$ pulsed oscillator and amplifying the resultant $f_{0}$ to $f_{1} \mathrm{Mc} / \mathrm{s}$ signal to provide the transmitter output.

The same variable oscillator is used as the local oscillator for a superheterodyne receiver of intermediate frequency $f_{x} \mathrm{Mc} / \mathrm{s}$. This receiver has an untuned input stage coupled directly to aerial, and is thus tuned at any instant to the transmitter frequency insuring automatic tracking.

\section{Design Requirements}

The various factors which must be considered in arriving at particular design features are considered below:

\subsection{Frequency Range}

The upper frequency limit is normally imposed by the maximum value of $F_{2}$ critical frequency. At sunspot maximum this may exceed $15 \mathrm{Mc} / \mathrm{s}$ but is seldom greater than $20 \mathrm{Mc} / \mathrm{s}$. This limit may be exceeded by the critical frequency of sporadic $E$ but this is exceptional and current research practice is to consider percentage of time $f_{0} E_{s}$ exceeds $5 \mathrm{Mc} / \mathrm{s}$.

Several factors influence the selection of a lower frequency limit. At very low frequencies elaborate transmitter and receiver antenna systems are necessary due to radiation inefficiency, and at frequencies below $1.5 \mathrm{Mc} / \mathrm{s}$ broadcast station interference in many areas causes considerable lack of resolution on the reproduced ionogram.

Many ionospheric research projects require detailed examination of a small portion of the frequency spectrum. It is desirable therefore, that the frequency range be variable within the limits discussed above. This also readily enables expansion 
or contraction of frequency range to accommodate sunspot maximum or sunspot minimum conditions.

\subsection{Scan Period and Scan Rate}

There is now reason to believe that almost continuous changes in ion density are occurring in the ionosphere, particularly in winter months when daylight records taken at intervals of 1 min often show marked differences from each other [Heisler, 1958]. In order, therefore, to prevent distortion and consequent misinterpretation of the record, particularly in deducing electron density profiles, scan period should be no greater than 15 sec.

Consequent deterioration of integrated signal noise ratio as indicated by Dieminger [1959] does occur but should preferably be improved by means other than increasing scan period, such as careful receiver design or by photographic integration as discussed in section 4.4.

The scan rate should be variable to allow several scans per minute in special studies e.g., sporadic- $E$ and a low rate for economical normal use.

\subsection{Scan Law}

The increasing use of ionograms in electron density profile analysis demands higher scaling accuracies than previously, particularly in regions where ion density changes slowly with height. Irrespective of accuracy of individual frequency calibration points therefore, interpolation between these points must be accurate. King [1960] has stressed the value of a logarithmic law scan in certain simplified $N(h)$ reduction techniques, and this type of law is also of value in ionospheric prediction analysis.

However, in practice the usual logarithmic law on many ionograms is only approximate and error can occur in interpolating between frequency marks. Interpolation is expedited by use of a linear scan law and small variations in this will usually introduce errors of less magnitude than those due to difficulties in interpolating an approximate logarithmic scale. One feature of log scan law is the open frequency scale at low frequencies where considerable accuracy is often required. However, this is usually at the expense of accuracy in reading $f_{0} F_{2}$.

\subsection{Transmitter Pulse Length and Receiver Bandwidth}

Since minimum detectable signal power is inversely proportional to pulse length [Herb and Sinsheimer, 1947], pulse lengths should be as long as possible consistent with required resolution. Price [1960] suggests that there is little advantage in pulse length $t$ being shorter than $100 \mu S$ due to loss of resolution by ionospheric dispersion. This argument, however, does not apply to sporadic $E$ ionization where a pulse length of $35 \mu \mathrm{S}$ would be required to resolve the $5 \mathrm{~km}$ stratification observed by Briggs [1958].

Pulse length is also a factor in determining receiver bandwidth and the optimum value is $1.2 / t \mathrm{Mc} / \mathrm{s}$ [Purcell, 1947].

\section{Design Principles}

The following design principles apply mainly to panoramic ionosondes. For purpose of detailed discussion the recorder may be considered to consist of the following main sections: variable frequency oscillator, fixed frequency oscillator, transmitter, receiver, and calibration system.

\subsection{Variable Frequency Oscillator (VFO)}

This oscillator provides a substantially constant output over the frequency range $\left(f_{0}+f_{x}\right) \mathrm{Mc} / \mathrm{s}$ to $\left(f_{1}+f_{x}\right) \mathrm{Mc} / \mathrm{s}$ in the scan period $T$ sec. Since the wide frequency spectrum output supplies energy to three individual circuits, namely transmitter mixer, receiver mixer, and frequency calibrator it is essential that oscillator output impedance be low. High voltage output is not necessary as receiver and calibrator require only a few volts for satisfactory operation, and for reasons discussed later in section 4.3a high level mixing in the transmitter mixer stage is difficult to achieve.

The rate of change of frequency $\left(f+f_{x}\right) \mathrm{Mc} / \mathrm{s}$ of the oscillator with time $t$ determines the scan law of the recorder, and this law is usually logarithmic or linear in form. If a rotating capacitor is used to vary the oscillator frequency it is possible to shape the rotor plate so as to give the desired rate of charge of capacitance with rotation angle. In practice, however, only an approximation to the required law is obtainable due to unpredictable edge effects between rotor and stator, and an exact law necessitates use of a rotating cam profile with a cam follower mounted on the capacitor shaft.

Any suitable rotating capacitor may be made to give a similar approximation to the required law by applying three point tracking techniques [Green, 1943] by addition of a series capacitor.

Electronic control of scan frequency may be obtained over a limited range by use of the recently developed voltage variable capacitor or Varicap [Hammerslag, 1959] or by application of a ring type resistance capacity feedback oscillator developed by Cormack [1951]. The frequency of this oscillator can be varied over a considerable range by application of a control voltage.

Electronic sweep control has the additional facility that any sweep range $f_{0}$ to $f_{1}$ can be obtained by suitable preset biasing arrangements while still maintaining scan law. A similar facility in mechanical scan arrangements can only be obtained by changing cam profiles. This form of control also removes the usual limitations on scan rates placed by mechanical sweep arrangements. Scans may be repeated as often as desirable, and minimum scan periods are limited mainly by possible receiver detuning before return of ionospheric echoes.

\subsection{Fixed Frequency Oscillator (FFO)}

This oscillates at a frequency of $f_{x} \mathrm{Mc} / \mathrm{s}$ and since the highly sensitive receiver in the recorder has a similar intermediate frequency, it is essential that 
the oscillator be pulse modulated by the transmitter keying pulse to prevent continuous receiver paralysis. It is extremely difficult even with most elaborate oscillator shielding to prevent leakage of signal into the receiver circuits.

The inherent accuracy of frequency calibration depends upon the accuracy of this oscillator. However the necessary requirements are not stringent and it is shown in section 5.5 that there is little advantage in having a fixed frequency oscillator accuracy greater than about one part in three thousand. This can be readily obtained with sufficient long term stability by means of a carefully constructed pulsed Hartley oscillator [Gamertsfelder and Holdam, 1949]. Pulse modulation of crystal oscillators is difficult and it is usually necessary to crystal control an oscillator at a submultiple frequency $f_{x} / n \mathrm{Mc} / \mathrm{s}$ and multiply this by pulsed amplifiers to give the required $f_{x} \mathrm{Mc} / \mathrm{s}$ output. Unfortunately there may be leakage of continuous $f_{x} \mathrm{Mc} / \mathrm{s}$ harmonic radiation into receiver circuits.

\subsection{Transmitter}

This consists essentially of a mixer to obtain the sweep frequency from the variable frequency oscillator output and the pulsed output of the fixed frequency oscillator, followed by a wide band amplifier feeding the transmitter aerial. These require a balanced output and hence push-pull output is usually employed, with the additional advantage of providing higher power output particularly in class $\mathrm{B}$ operation. Adoption of this necessitates some form of phase inversion in the amplifier chain to provide out of phase driving voltages for the output stage. In order to obtain sufficient gain with a minimum of tubes, wide band coupling techniques must be used throughout mixer and amplifier circuits. The various approaches which can be made to these problems are considered below.

\section{a. Resistance Capacitance Coupling}

In this type of coupling the top frequency to be amplified $f_{1}$ is approximately equal to bandwidth $B_{n}$. If the amplifier consists of $n$ stages and if $B$ is the bandwidth of each stage

$$
B_{n}=\frac{B}{\sqrt{n}}
$$

The normal maximum plate resistor $R$ in any stage is given by

$$
R=\frac{1}{2 \pi B C}
$$

where $C$ is the interstage shunt capacity.

$R$ may be increased by a factor of 1.77 to 2.4 by the use of two and four terminal network coupling techniques, described by Walker and Wallman [1948]. The total interstage capacity $C$ should be measured as strays are unknown and published values of tube capacitances are cold values and have been found to deviate considerably from hot tube values.
Some resistors have been found to change value considerably at high frequencies, and $R$ for preference should be deposited carbon on a ceramic former.

It is possible using network coupling techniques to deliberately peak the high frequency gain of an individual stage [Walker and Wallman, 1948]. If this is done then bandwidth shrinkage as expressed by eq (1) is not so severe, and bandwidth requirements for an individual stage may be relaxed.

The limitations imposed by maximum power rating of tubes may be overcome by pulse switching stages for the duration of the transmitter pulse. Screen voltages may then be increased by a factor $N$ with consequent increase in mutual conductance $g_{m}$ to $\sqrt{N} g_{m}$ approximately. Moreover the effective grid base of a tube is lengthened by a factor $N$ approximately so that higher permissible grid voltage swings become possible, an important feature in high level stages.

One immediate consequence of pulse switching is that a large negative pulse appears across plate resistor $R$ due to change in plate potential level when the valve becomes operative, and small coupling time constants consistent with adequate coupling at frequency $f_{0}$ are necessary to prevent appearance of this on the grid of the following stage.

The valve chosen as transmitter mixer must have a high value of $g_{m}$ since conversion transconductance is approximately $g_{m} / 3$. This restricts choice of mixer valves to those with a relatively short grid base. Consequently high voltage inputs are not possible and high level mixing is difficult to achieve.

\section{b. Wide Band Transformer Coupling}

One difficult problem associated with the transmitter design is to provide sufficient power output into a low impedance. In practice the push-pull output stage is directly coupled to the aerial circuits to develop power in an effective load of $600 \mathrm{ohms}$ under class A operating conditions. The type of tube chosen as final amplifier must therefore have high mutual conductance, low plate resistance and large negative grid voltage for plate current cutoff, conditions which are most readily met by pulse modulator tetrodes specially developed for radar purposes.

Performance can be improved by matching aerial impedence to the plate resistance of the final amplifiers by means of a wide band transformer. This enables class B operation to be used with its much higher plate efficiency and provided sufficient power is available from the previous stage, grids may be driven positive, consequently valves need not have a high value of grid voltage cutoff. This relaxation in required characteristics permits use of normal high perveance valves and effects considerable economy.

A complete discussion of very wide band transformer design is given by Maurice and Minns [1947]. The upper frequency limit is mainly controlled by leakage inductance which is minimized by keeping number of turns low and careful attention to physical design. At low frequencies number of turns must 
be sufficient to provide required inductance, and is reduced by using a core of modern powdered iron dust materials possessing high initial permeability. However, care must be taken to insure that flux saturation does not occur at the power outputs considered. If the transformer has balanced windings, then a high degree of balance can only be obtained by inserting an electrostatic shield between windings.

\section{c. Phase Inversion}

If the transmitter has push-pull output, some form of phase inversion is necessary in the amplifier chain to provide driving voltages $180^{\circ}$ out of phase. If the phase splitter is used in the early stages of the amplifier then all subsequent stages must be push pull in form, with consequent duplication of valves, increase in power consumption and the risk of voltage unbalance occurring at the output stage grids. When the phase splitter is placed in late stages of the amplifier, it may be necessary to employ large pulse tetrodes.

A convenient means of obtaining the necessary inversion is by using an amplifier having unity gain as described by Heisler [1955], or the cathode coupled unbalance to balance system used in the C4 recorder [Brown, 1959].

Another method of realizing the necessary antiphase voltages is by use of a broad band transformer with a balanced secondary winding. This can be used to advantage as a means of coupling the driver stage to power output stages operated under class B conditions.

\section{d. Transmitter Pulser}

This applies a large pulse of suitable polarity to either grid or screen circuits rendering the valve operative for a period of $t \mu \mathrm{sec}$. During this period the pulser must present a low impedance to the appropriate circuits of the controlled valves. This permits loading by complex impedences in pulsed grid circuits and enables pulsed screen circuits to be operated without RF bypass capacitors, both factors impairing pulse shape, and inhibiting feedback between common pulsed stages.

In practice the bootstrap type pulser [Glasoe, 1948] pulsing screen grids has been found most satisfactory.

\section{e. Transmitter Aerial}

Effective power output of the transmitter depends to a large extent on the radiation efficiency of the associated antenna system. The broadband requirements demand design compromise particularly at the low frequency end, where physical dimensions of the antenna have to be at least a quarter of a wavelength for efficient radiation. Usually a $600-o h m$ delta type antenna is employed similar to that described by Cones et al. [1950]. Unfortunately, considerable power is dissipated in the terminating resistance and at low frequencies as much as 80 percent of the power input to the aerial may be lost in this manner [Cones, 1951]. Where research is concerned with this end of the spectrum, it may be necessary to provide high power input to the aerial system to compensate for this.

There appears to be considerable need for development of suitable aerial designs for this purpose, particularly with a view to maintaining aerial efficiency at low frequencies.

\subsection{Receiver}

This consists of an untuned aerial stage and mixer followed by sufficient intermediate frequency amplification, to provide several volts of signal at the detector with high signal noise ratio. Since all frequencies arriving at the aerial appear on the mixer grid, it is necessary to attenuate frequencies outside the signal band $f_{0}$ to $f_{1} \mathrm{Mc} / \mathrm{s}$ to prevent serious cross modulation. In practice a high pass filter is inserted in aerial circuits to remove broadcast station interference below $f_{0}$, while a resonant trap tuned to $f_{\mathrm{x}} \mathrm{Mc} / \mathrm{s}$ prevents breakthrough at the intermediate frequency of the receiver.

A similar restriction of pass band may be obtained by using a well designed wide band transformer on aerial input circuits. Aerial input is usually balanced in form and use of a balance to unbalance transformer greatly simplifies front end design.

Bandwidth requirements are mentioned in section 3.4. Intermediate frequency $f_{\mathrm{x}}$ is usually greater than $20 \mathrm{Mc} / \mathrm{s}$, hence gain and selectivity can be improved by using double frequency conversion. As will be described later the frequency calibration system uses a $1 \mathrm{Mc} / \mathrm{s}$ harmonic generator; the value of second intermediate frequency should therefore be other than integral to prevent interference from this source.

The amplitude of the received pulses covers a large dynamic range and if receiver gain is adjusted to record weak echoes satisfactorily, strong echoes invariably saturate the receiver with consequent loss of complex splitting detail.

This effect may be minimized by some form of instantaneous automatic gain control [Lawson and Uhlenbeck, 1948a], or alternatively by some form of logarithmic receiver such as that described by Chambers and Page [1954], which has a logarithmic gain response over a very wide range of amplitude of input signals. The latter in particular is most suitable for measurement of absolute amplitudes in ionospheric absorption research applications.

Automatic gain control is particularly effective in discriminating against unwanted $\mathrm{CW}$ signals. Simlar discrimination by differentiation of the received echo before application to display should be used with caution or loss of complex splitting detail in the received echo may occur. The usual RC filter is not satisfactory [Lawson and Uhlenbeck, 1950b] and a high pass filter with low frequency cutoff $1 /(2 t)$ should be used. The technique is only of real value if intermediate frequency amplifiers are not overloaded [Haworth and Jordon, 1947].

Receiver output should provide echoes of negative polarity for application to the grid of the cathode ray display tube. Photographic integration on the resultant ionogram has been shown to provide considerable noise discrimination. 


\subsection{Calibration System}

The final ionosonde presentation must be provided with calibration of both frequency and height. Frequency calibration is usually provided by heterodyning the variable frequency oscillator output $f_{0}+f_{x}$ to $f_{1}+f_{x} \mathrm{Mc} / \mathrm{s}$ with harmonics from a $1 \mathrm{Mc} / \mathrm{s}$ harmonic generator and using the resultant zero beats to generate short sharp pulses corresponding to each megacycle in the range $f_{0}$ to $f_{1}$. These pulses are then applied to intensifier circuits in such a way to provide suitable marks on the visual display.

The generator is usually crystal controlled and accuracy of individual marks depends mainly on accuracy of $f_{x}$. However, since the received echo falling on a given frequency mark includes a number of frequencies in the optimum receiver bandwidth, it is doubtful whether frequencies read to any greater accuracy than one half of this have any real significance. This demands a FFO stability of 1 part in 3,000 .

Height calibration appears on the final display as a series of sharp marks corresponding usually to $50 \mathrm{~km}$ height separation. These are generated by a 3,000 cycle oscillator gated so that it only operates during each height display.

An upper limit to overall accuracy of height calibration of at least $1 \mathrm{~km}$ is imposed by errors in time measurement due to rise time of received echoes and variations of receiver delay with signal amplitude [Chance, 1949]. The maximum height range usually employed is $1,000 \mathrm{~km}$, and therefore accuracy is limited to one part in 1,000. This order of stability is easily attained in a well designed RC oscillator.

\section{Recorder Description}

An ionosonde is described which covers the frequency range 1 to $20 \mathrm{Mc} / \mathrm{s}$. This has been constructed in the laboratory of the Radio Research Board Sydney, based on many of the design techniques discussed above. The complete recorder weighs approximately $150 \mathrm{lbs}$ and is contained in a $22 \mathrm{in}$. square cubicle which stands 34 in. high (without camera and camera boxes). The ionosonde features complete electronic operation, with a nominal scan period of 15 sec repeated at a scan rate of 1 per minute, with a linear or logarithmic scan law. However, scan period can be made as short as 2 sec and this can be repeated almost continuously. Nominal frequency range is 1 to $20 \mathrm{Mc} / \mathrm{s}$ but any part of this range may be selected by the setting of two controls. A manual control permits a manual scan to be made, or if necessary the ionosonde may be set to operate on any particular fixed frequency. Transmitter pulse length is variable between 40 and $100 \mu$ sec and the pulse peak power input to a 600 ohm balanced antenna averages $3 \mathrm{kw}$ at a pulse repetition frequency of $50 \mathrm{c} / \mathrm{s}$. Most of the power supplies are electronically voltage regulated. The complete recorder can be constructed for less than $£ 800$ (Australian).
Because of space considerations it is obviously impossible to publish complete circuit diagrams or to give detailed technical information. The following description therefore lists briefly the most important technical features of the equipment.

\subsection{Variable Frequency Oscillator (VFO)}

This is developed from a circuit due to Cormack [1951]. Basically the circuit is a resistance capacitance ring type oscillator, in which the necessary phase shift is provided by a triode amplifier and four cathode followers. Phase rotation and hence oscillator frequency is controlled by varying the mutual conductance of the cathode followers with an applied voltage. The voltage developed by the oscillator is from 1.5 to 1.0 volts rms over the frequency range 29 to $48 \mathrm{Mc} / \mathrm{s}$ and the almost linear frequency range shift for applied control obtained is amplified to supply 4.0 to 6.0 volts rms to the transmitter and receiver mixers. The transmitter mixer is supplied directly from this amplifier while the receiver mixer and frequency calibrator mixer are coupled to the amplifier by cathode followers.

\subsection{Fixed Frequency Osciliator (FFO)}

This is a pulsed Hartley oscillator as described by Gamertsfelder and Holdam [1949a] in their figure 4.45 . The oscillator tube operates over a linear region of the tube characteristics and is therefore free from frequency deviation effects due to stray power amplifier pulses in the grid circuits. C class pulsed oscillators are particularly prone to this. A buffer amplifier is used after the oscillator to drive the transmitter mixer cathode.

\subsection{Transmitter}

A type $12 \mathrm{BY} 7$ tube was chosen for the transmitter mixer from several types tried. The VFO voltage is injected into the control grid and the FFO voltage into the cathode circuit. The plate circuit is coupled to a 6CK6 amplifier by a four terminal network with a cutoff frequency of $22 \mathrm{Mrc} / \mathrm{s}$, which inhibits higher order component frequencies from reaching the $6 \mathrm{CK} 6$ grid. This amplifier is similarly coupled to a type 6CM5 amplifier which is transformer coupled to the power amplifiers. The transformer is a wide bandwidth type wound on a toroid A4 Ferroxcube core, providing out of phase voltages to drive the final push-pull amplifiers. These are type 6CM5 tubes coupled to antenna circuits by another wide bandwidth transformer. All amplifiers are screen grid pulsed by a common bootstrap type pulser unit.

\subsection{Receiver}

Type $6 \mathrm{BX} 6$ tubes are used as $\mathrm{RF}$ amplifiers and these are coupled to type $6 \mathrm{EJ} 7$ tubes as mixers by four terminal coupling networks. The variable oscillator voltage of average value $1.8 \mathrm{v} \mathrm{rms}$ is 
injected into the cathode circuits of the mixer tubes. The mixer stage is followed by a single stage of IF amplification at $28 \mathrm{Mc} / \mathrm{s}$ which is then converted to $1.9 \mathrm{Mc} / \mathrm{s}$ and amplified by two additional stages. Dynamic range is improved by use of variable MU tubes throughout the amplifier and application of automatic gain control to the last stage. This is followed by a conventional compensated diode detector and one stage of video and audio amplification. The receiver has an overall gain of $114 \mathrm{db}$ and a bandwidth of $30 \mathrm{kc} / \mathrm{s}$.

\subsection{Calibration System}

This provides suitable range marks and frequency marks on the final display. The height calibrator uses a gated resistance capacity $15 \mathrm{kc} / \mathrm{s}$ oscillator and this signal is divided to provide a $3 \mathrm{kc} / \mathrm{s}$ signal. The $15 \mathrm{kc} / \mathrm{s}$ and $3 \mathrm{kc} / \mathrm{s}$ signals are clipped, suitably differentiated, mixed and applied to the display to produce fine $10 \mathrm{~km}$ and broad $50 \mathrm{~km}$ height marks. Either or both calibrations may be selected at will.

The frequency calibrator consists of a $6 \mathrm{BL} 8$ tube as a triode $1 \mathrm{Mc} / \mathrm{s}$ crystal oscillator and pentode harmonic generator. This output is mixed with the output of the variable frequency oscillator in the pentode section of a $6 \mathrm{AJ} 8$ tube and the resulting heterodyne beats which correspond to $1 \mathrm{Mc} / \mathrm{s}$ frequency intervals are amplified, squared and applied to a multivibrator which when operative, removes the time base synchronizing signal and hence the cathode ray tube display. Special provision is made to insure that frequency intervals always occupy the same deflection length on the display irrespective of duration of scan period, and a later improvement places frequency markers on top and bottom of the record only.

\subsection{Control Unit}

The control unit provides the basic variable $d-c$ voltage to vary the frequency of the VFO. The same voltage is used to provide display tube deflection when a linear scan law is desired. An adjustable RC charging circuit with suitable controls to vary amplitude and initial starting voltage establishes start and finish of scan voltage and hence the particular frequency scan. A synchronous motor is used to provide regular $1 \mathrm{~min}$ or longer intervals between scans.

\subsection{Display}

Two displays are provided, one is on the face of a 5 in. long persistence screen cathode ray tube and is used for monitoring, while the second display is on a similar tube having a normal persistence screen which is photographed with either a $35 \mathrm{~mm}$ or $16 \mathrm{~mm}$ camera.

Typical positive prints of ionograms produced by the recorder are shown in figures 1,2, and 3 . Figures 1 and 2 are both examples of 12 sec sweeps. In figure 1 the frequency range covered is from 1 to 14 $\mathrm{Mc} / \mathrm{s}$, while in figure 2 the frequency range is 2 to $20 \mathrm{Mc} / \mathrm{s}$. Figure 3 shows a very fast sweep of $2 \mathrm{sec}$ over the frequency range 0.1 to $12 \mathrm{Mc} / \mathrm{s}$. It will be noticed that the sweep is so rapid that individual strokes of the time base are obvious, yet in spite of this the amount of information available does not differ materially from that in figures 1 and 2 . The examples serve to illustrate the great versatility of the equipment both in scan period and frequency range.

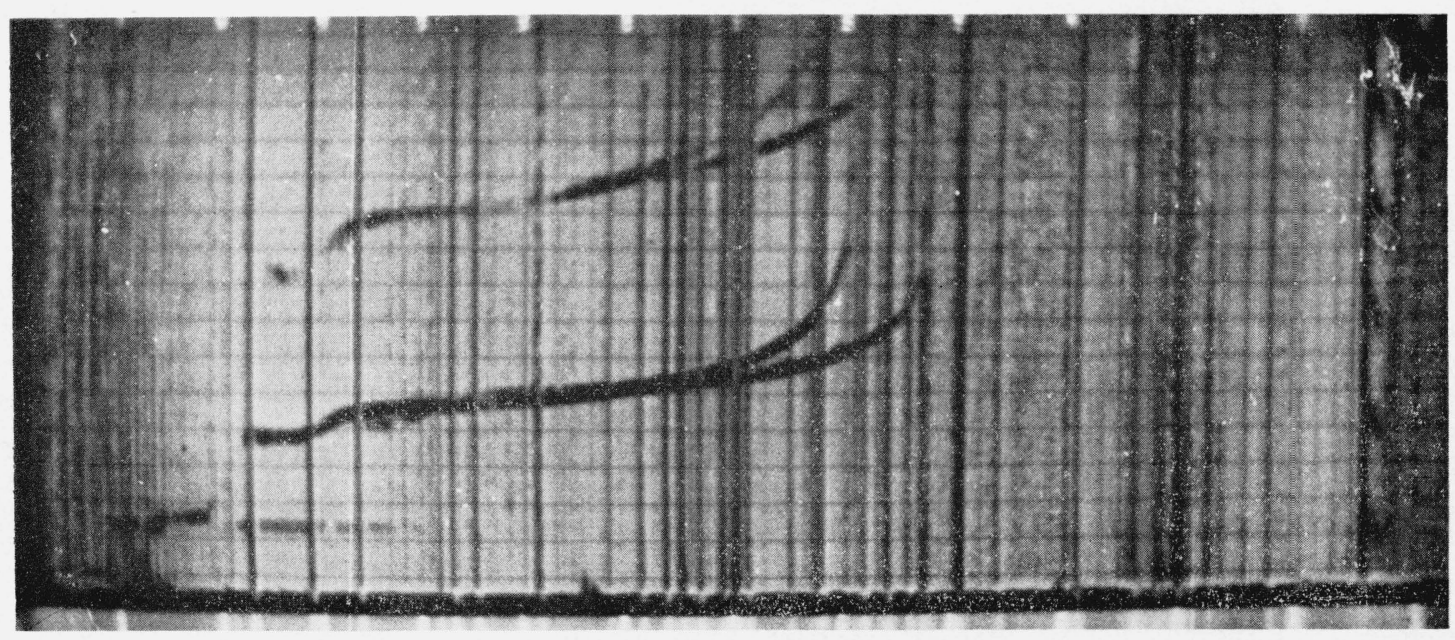

FIGURE 1. Typical ionogram covering the frequency range 1.0 to $14 \mathrm{Mc} / \mathrm{s}$ in a scan period of $12 \mathrm{sec}$. 


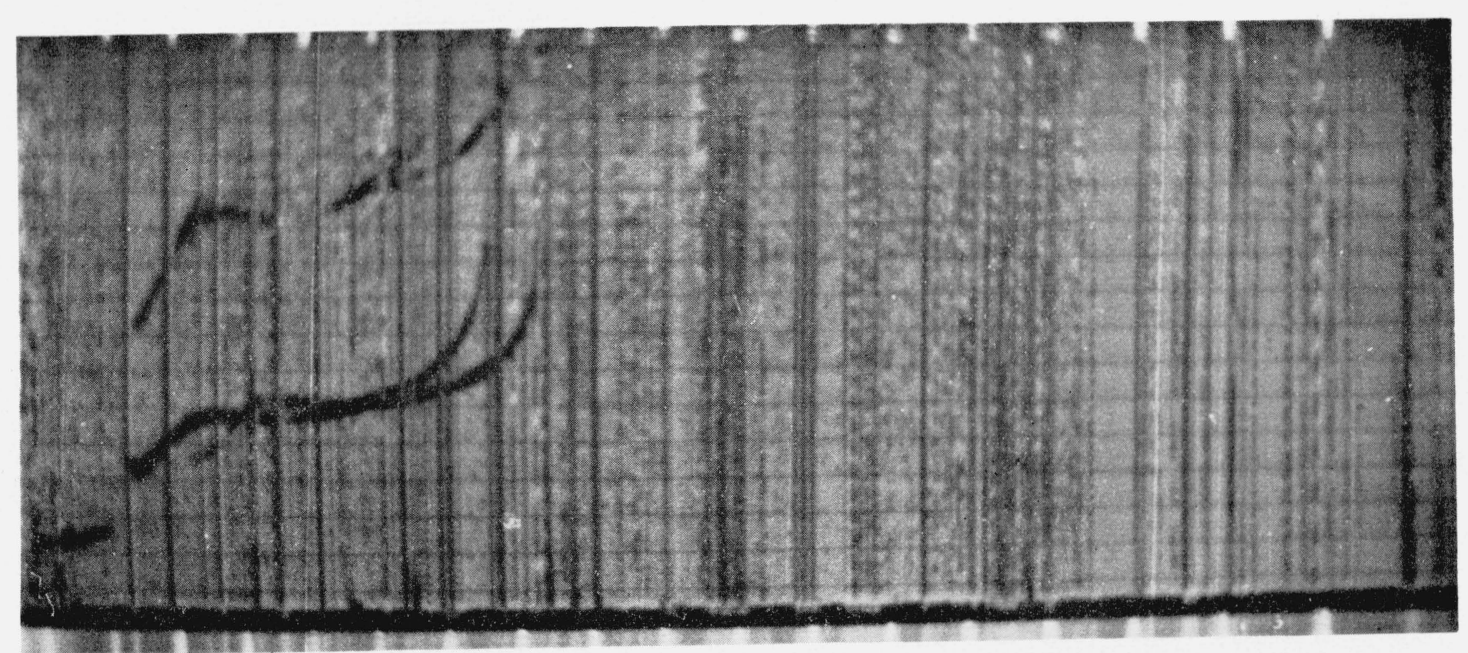

Figure 2. Typical ionogram covering the frequency range 2.0 to $20 \mathrm{Mc} / \mathrm{s}$ in a scan period of $12 \mathrm{sec}$.

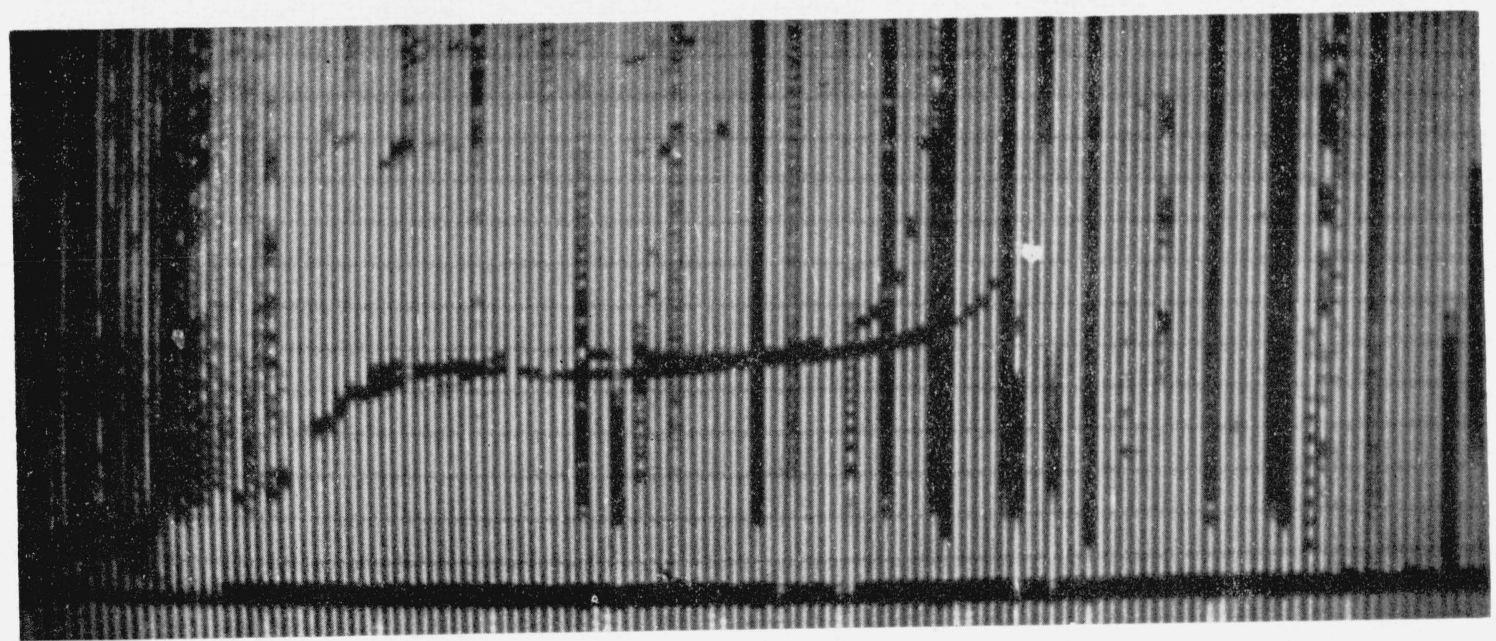

FIGURE 3. Typical ionogram covering the frequency range 1.0 to $12 \mathrm{Mc} / \mathrm{s}$ in a scan period of $2 \mathrm{sec}$

\section{Conclusions}

Many facets of panoramic ionosonde design have been considered. There are possibly other design. features, particularly for specific research projects which have not been included, but it is felt that the factors discussed constitute enough information to provide a basic ionosonde design. Some of the main design features emphasized are as follows:

(i) The use of electronic scan control provides many desirable ionosonde features economically. Rapid scans over the whole or any part of the frequency range are possible, and scan laws either logarithmic or linear can be selected at will.

(ii) Considerable economy can be effected in transmitter circuits by the use of wide band transformer coupling. (iii) There is little advantage in using transmitter pulse lengths less than $100 \mu \mathrm{sec}$, except for $E_{s}$ investigations where a pulse length of $40 \mu$ sec may be beneficial.

(iv) Present transmitter antenna systems are very inefficient at low frequencies. There is need for research into suitable aerials for this particular purpose.

(v) To avoid technical design difficulties, the frequency range of the ionosonde should not be greater than necessary. A range of 1.0 to $20 \mathrm{Mc} / \mathrm{s}$ is suggested.

(vi) The scan period should not exceed $15 \mathrm{sec}$

(vii) The scan rate should be frequent at least one frame per minute, although this of necessity is governed by economy in film consumption.

(viii) Differentiation of receiver output is advantageous, only if intermediate fre- 
quency stages are not overloaded and care is taken in requisite filter design.

(ix) Considerable noise discrimination is obtained if the signal applied to display cathode ray tube grid is of negative polarity.

(x) There is some doubt as to the advantage of a logarithmic scan. law, and interpolation. errors in such a case are likely to be greater than those occurring in a similar linear law. Electronic scan control is particularly adaptable to generation. of a linear scan law.

(xi) For most purposes a receiver bandwidth of $30 \mathrm{kc} / \mathrm{s}$ is more than adequate even. for scan periods of the order of a few seconds.

A complete technical description will be given in a later paper.

This work has been carried out in the Electrical Engineering School of the University of Sydney as part of the research program sponsored by the Radio Research Board of the Commonwealth Scientific and Industrial Research Organisation.

We express our appreciation to the University of Sydney and in particular to Professor W. N. Christiansen for provision of facilities in the Electrical Engineering School. The authors also thank Dr. G. H. Munro for helpful discussions during the preparation of the paper.

\section{References}

Briggs, B. H., Some characteristics of sporadic-E ionisation as determined by a rapid frequency sweep experiment, Agardograph 34, sporadic-E ionisation, 151-161 (NATO advisory group for aeronautical research and development, 1958).

Brown, J. N., Automatic sweep-frequency ionosphere recorder Model C-4, Proc. IRE 47, No. 2, 296-300 (Feb. 1959).

Chambers, T. H. and I. H. Page, The high-accuracy logarithmic receiver, Proc. IRE 42, No. 8, 1307-1314 (1954).

Chance, B., Electronic time measurements, 1st ed., Radiation Laboratory Series, Vol. 20, ch. 3, 39-41 (McGrawHill Book Co. Inc., New York, N.Y., 1949).

Cones, H. N., Effect of a metal mast and guy wires on the performance of a $600 \mathrm{ohm}$ multiple-wire delta antenna, J. Research NBS 46, No. 2, 113-120 (Feb. 1951).

Cones, H. N., H. V. Cottony, and J. M. Watts, A $600 \mathrm{ohm}$ multiple-wire delta antenna for ionospheric studies, J. Research NBS 44, 475-488 (May 1950).

Cormack, A., Wide range variable-frequency oscillator, Wireless Engr. 28, No. 336, 266-270 (Sept. 1951).

Dieminger, W., Transient fine structure of the $E$ layer, J. Atmospheric and Terrest. Phys. 16, Nos. 1/2, 179 (Oct. 1959).
Gamertsfelder, G. T., and J. V. Holdam, Waveforms, 1st ed., Radiation Laboratory Series, Vol. 19, ch. 4, 143-145 (McGraw-Hill Book Co., Inc., New York, N.Y., 1949).

Glasoe, G. N., Pulse generators, 1st ed., Radiation Laboratory Series, Vol. 5, ch. 4, 120 (McGraw-Hill Book Co., Inc., New York, N.Y., 1948).

Green, A. L., Superheterodyne tracking charts III, The padded signal circuit, Wireless Engr. 20, No. 243, 581-594 (Dec. 1943)

Hammerslag, J., Circuit design using silicon capacitors, Electronics 32, No. 38, 48-50 (Sept. 18, 1959).

Haworth, L. J. and W. H. Jordon, Radar System Engineering, 1st ed., Radiation Laboratory Series, Vol. 1, ch. 12, 459 (McGraw-Hill Book Co. Inc., New York, N.Y., 1947).

Heisler, L. H., A panoramic ionospheric recorder for the study of travelling disturbances in the ionosphere, Australian J. Appl. Sci. 6, No. 1, 1-6 (Jan. 1951).

Heisler, L. H., Anomalies in ionosonde records due to travelling ionospheric disturbances, Australian J. Phys. 11, No. 1, 79-90 (Jan. 1958).

Heisler, L. H. and J. D. Whitehead, F-region travelling disturbances and sporadic- $E$ ionisation, J. Geophys. Research 65, No. 9, 2767-2773 (Sept. 1960).

Herb, R. G., and R. L. Sinsheimer, Radar system engineering, 1st ed., Radiation Laboratory Series, Vol. 1, ch. 15 596 (McGraw-Hill Book Co. Inc., New York, N.Y., 1947).

Kelso, J. M., The determination of the electron density distribution of an ionospheric layer in the presence of an external magnetic field, J. Atmospheric and Terrest. Phys. 5, No. 1, 11-27 (Mar. 1954).

Kelso, J. M., The calculation of ionospheric electron density distributions, J. Atmospheric and Terrest. Phys. 10, No. 2, 103-109 (Feb. 1957).

King, G. A. M., Use of logarithmic frequency spacing in ionogram analysis, J. Research NBS 64D, No. 5, 501-504 (Sept.-Oct. 1960).

Lawson, J. L., and G. E. Uhlenbeck, (a) Threshold signals, 1st ed., Radiation Laboratory Series, Vol. 24, ch. 11, 303306 (McGraw-Hill Book Co., Inc., New York, N.Y., 1950).

Lawson, J. L. and G. E. Uhlenbeck, (b) Threshold signals, 1st ed., Radiation Laboratory Series, Vol. 24, ch. 12, 342343 (McGraw-Hill Book Company Inc., New York, N.Y., 1950).

Maurice, D., and R. H. Minns, Very wide band radio frequency transformers, Wireless Engineer 24, Nos. 285 and 286, 168 177, 209-216 (June and July 1947).

Munro, G. H., Travelling disturbances in the ionosphere, Proc. Roy. Soc. A 202, 208-223, 1950.

Price, W. L., (1960) private communication.

Purcell, E. M., Radar system engineering, 1st ed., Radiation Laboratory Series, Vol. 1, ch. 2, 33-35 (McGraw-Hill Book Co., Inc. New York, N.Y., 1947).

Schmerling, E. R., and C. A. Ventrice, Coefficients for the rapid reduction of $P^{\prime}-f$ records to $N-h$ profiles without computing aids, J. Atmospheric and Terrest. Phys. 14, Nos, 3/4, 249-261 (June 1959).

Sulzer, P. G., Sweep frequency ionosphere equipment, J. Appl. Phys. 20, No. 2. 187-196 (Feb. 1949).

Walker, R. M., and H. Wallman, Vacuum tube amplifiers, 1st ed., Radiation Laboratory Series, Vol. 18, ch. 2, 75-79 (McGraw-Hill Book Co., Inc., New York, N.Y., 1948).

(Paper 65D6-165) 